\section{Clinical picture, diagnosis and treatment of rosacea, complicated by Demodex mites}

\author{
Alexey Kubanov, Yuliya Gallyamova, \\ Anzhela Kravchenko \\ Russian Medical Academy of \\ Continuous Professional Education, \\ Ministry of Healthcare of the Russian \\ Federation, Moscow, Russia
}

\begin{abstract}
The article analyzes the clinical picture and course of rosacea in patients with Demodex mites. It presents the advantages of using the method of confocal laser scanning microscopy over the method of light microscopy of facial skin scrapes. The aimes were to study the influence of Demodex mites on the clinical picture and course of rosacea; to compare laboratory and instrumental diagnostic methods for detecting Demodex mites; to evaluate the effectiveness of external therapy aimed at eliminating Demodex mites. 212 people were examined. The study included healthy patients, patients with a diagnosis of rosacea with the presence and absence of Demodex. The presence of Demodex mites was confirmed by two methods of study (light microscopy of skin scrapes and confocal laser scanning in vivo microscopy). Demodex mites promote the development of acute-inflammatory morphological elements, increase the duration of the condition (more than 5 years, $\mathrm{P}<0.01$ ) and the probability of recurrence (from 1 to 3 relapses in $39.5 \%$ of patients, $\mathrm{P}<0.05$ ), resulting in a decrease in the quality of life of patients (dermatology life quality index is $12.5 \pm 4.5, \mathrm{P}<0.05)$. Antiparasitic drug ivermectin, in the form of an external form, at a concentration of $1 \%$ has a high therapeutic efficacy (in $93.3 \%$ of cases). Demodex folliculorum shows signs of parasitism, while Demodex folliculorum brevis is a saprophyte. The severity of the condition does not depend on the quantitative load of the mites in the scrape. As an antiparasitic drug, it is recommended to use $1 \%$ ivertmectin.
\end{abstract}

\section{Introduction}

In connection with the growth of cultural level of the society as a whole and the increase in individual exactingness to one's own appearance, both among men and women, face dermatosis remain one of the pressing problems. Despite the large num- ber of scientific works devoted to the pathogenesis of rosacea, the question of Demodex mites' role in the development of the clinical picture of the condition remains open in the contemporary literature.

According to some authors, Demodex mites are representatives of the conditionally pathogenic microflora of facial skin along with Propionibacterium acnes, Staphylococcus epidermidis and Malassezia fungi. ${ }^{1,2}$ This opinion is supported by the fact that in $55-100 \%$ of cases, mites are detected, both in patients with face dermatosis and with patients having no clinical signs of dermatological illnesses. ${ }^{3-5}$ However, there are scientific papers proving that Demodex mites are capable of pathogenic parasitization and are the most frequently detected microbial agents in rosacea. ${ }^{4}$ At the same time, attention is drawn to the lack of Demodicosis diagnosis in the International Classification of Illnesses of $\mathrm{X}$ revision, which points to the fact that Demodex mites species act rather as an agent complicating the course of rosacea. Currently, two species of Demodex mites parasitize on human skin: Demodex folliculorum and Demodex brevis. ${ }^{6}$ The modern literature has no substantiated scientific studies indicating the role of the species belonging of the causative agent in the formation of the clinical picture of rosacea. Existing assumptions are not fully proven. The available data on the parasitization of Demodex mites in patients with rosacea are inconsistent and, in many cases, are mutually exclusive.

The available diagnostic methods for detecting Demodex mites do not meet the requirements of modern medicine, do not guarantee the absolute reliability of the test results, and are often traumatic. One modern diagnostic technique in dermatology is 25 lasers scanning in vivo microscopy. ${ }^{7}$ This is an innovative method, the advantages of which are non-invasiveness and high information content, however, to date, in the Russian Federation this method has not been used to detect Demodex mites. Thus, in order to improve the quality of diagnosis and therapy, it becomes necessary to conduct a scientific study with an analysis of the clinical picture, comparing the methods of diagnosis and treatment of patients with rosacea associated with Demodex mites.

The purpose of the study is to evaluate laboratory and instrumental diagnostics and therapy of patients with rosacea complicated by Demodex mites.

\section{Objectives of the study}

i) To study the influence of Demodex mites on the clinical picture and course of rosacea;
Correspondence: Yuliya Gallyamova, Russian Medical Academy of Continuous Professional Education, Ministry of Healthcare of the Russian Federation, 123995, 2/1 Barrikadnaya Street, Moscow, Russia.

E-mail: yulya.gallyamova.69@mail.ru

Key words: Dermatology life quality index; Demodex mites; Demodex folliculorum; Demodex folliculorum brevis.

Contributions: $\mathrm{AKu}$ and $\mathrm{YG}$ conceived the present idea, planned the experiments and supervised the findings of this work. AKr performed the computations and verified the analytical methods. All authors together investigated all aspects of the influence of Demodex mites on rosacea patients, discussed the results and contributed to the final manuscript.

Conflict of interest: the authors declare no potential conflict of interest.

Funding: none.

Received for publication: 11 March 2018. Revision received: 19 June 2018.

Accepted for publication: 25 July 2018.

This work is licensed under a Creative Commons Attribution-NonCommercial 4.0 International License (CC BY-NC 4.0).

(C) Copyright A. Kubanov et al., 2019 Licensee PAGEPress, Italy

Dermatology Reports 2019; 11:7675 doi:10.4081/dr.2019.7675

ii) To study the features of the clinical picture of rosacea associated with Demodex mites, depending on the species belonging to Demodex mites;

iii) Evaluate the effectiveness of laboratory and instrumental diagnostic methods for detecting Demodex mites;

iv) To evaluate the effectiveness of external therapy aimed at eliminating Demodex mites in patients with rosacea.

\section{Materials and Methods}

The work was performed at the databases of the Department of Dermatovenereology and Cosmetology of the Federal State-Funded Educational Institution Continuing Professional Education "Russian Medical Academy of Postgraduate Education" of the Ministry of Health of the Russian Federation and the Federal Government Budgetary Institution "Government Research Centre of Dermatovenereology and Cosmetology" of the Ministry of Health of the Russian Federation from 2013 to 2016 years. The 
study protocol was approved of the Ethical Committee of the Russian Medical Academy of Continuous Professional Education. Each participant was familiarized and signed the Informed consent of the participant in biomedical research.

During the study, a total of 212 people (men and women) were examined. The study included healthy patients, patients with a diagnosis of rosacea with the presence and absence of Demodex mites on the facial skin. During the study, three groups of patients and healthy people over the age of 18 were formed. I group - patients with a diagnosis of rosacea with the presence of Demodex mites. Patients were included in group I, in which the presence of Demodex mites was confirmed by two methods of study (light microscopy of skin scrapes and confocal laser scanning in vivo microscopy in an amount of more than 5 individuals per $1 \mathrm{~cm}^{2}$ ). II group is a comparison group, which was composed of patients with a diagnosis of rosacea with no Demodex mites. In Group II patients, two methods of study of Demodex mites were not found. III group comprises a comparison group, which included healthy people. Due to the fact that in 20 patients with rosacea, Demodex mites were detected by only one research method, they were did not include to the study, but the data of this group were used for statistical processing when comparing the effectiveness of diagnostic methods for the presence of Demodex mites.

\section{Methods of the study}

Anamnesis was collected and questionnaire survey according to the questionnaire of the Dermatology Life Quality Index, which included 10 items was filled by every participant.

Clinical: inspection and establishment of a preliminary diagnosis. The diagnosis of rosacea was established based on the clinical picture of the condition. To determine the severity of rosacea guided classification of the national rosacea society. ${ }^{8}$ All patients diagnosed with rosacea were counted morphological elements on the entire surface of the facial skin. Laboratory: i) Determination of the presence and species affiliation of Demodex mites with the help of light microscopy of skin scrapes, the contents of the sebaceous glands, hair follicles of the eyelashes and/or eyebrows, counting of the detected individuals, larvae, eggs per unit area $\left(1 \mathrm{~cm}^{2}\right)$; ii) Determination of the $\mathrm{pH}$ level of the facial skin is made as follows. Instrumental: i) Photographing patients before and after treatment; ii) Study of the facial skin with the help of confocal laser scanning in vivo microscopy to determine the presence of Demodex mites. Statistical: statistical processing of data was carried out by the packages of Microsoft Excel 2013 and SPSS 21. The interconnection of categorical indicators was established by Fisher's exact method. Fisher's exact test is a statistical significance test used in the analysis of categorical data when sample sizes are small. To assess the significance of the differences in the follicles, single-factor analysis of variance was used with paired comparisons. To assess the significance of the differences in the absence of a normal distribution, the Mann-Whitney test, the Kruskal-Wallis multiple comparison test, were used. Differences were considered significant at $\mathrm{P}<0.05$.

\section{Results}

\section{Clinical characteristics of patients included in the study}

A total of 192 respondents aged 18 to 79 (mean age $30.0 \pm 11.9$ ) were under supervision. There were 82 male participants and 110 female participants. The diagnosis of rosacea was exposed to 120 patients.

According to the method of the study, the patients were divided into two groups. Group I included patients with rosacea (60 people) who had a Demodex mite detected by two methods of study: laboratory - light microscopy of scrapes and instrumental confocal laser scanning in vivo microscopy in an amount of more than 5 individuals per $1 \mathrm{~cm}^{2}$, the second group included rosacea patients (60 people) who had a negative analysis for the presence of Demodex mites (Table 1). When comparing the data of the anamnesis of Groups I and II, the factors statistically significantly more frequent in patients with rosacea associated with Demodex mites were found. Statistical processing of data revealed that the frequency of detection of Demodex mites significantly differs depending on the factor triggering the development of the condition

Table 1. The distribution of patients by sex, age, diagnosis and the presence of Demodex mites.

$\begin{array}{lccc} & \text { Group I (with the presence of Demodex mites) } & \text { Total ( } \mathrm{n} ; \%) \\ \text { Sex } & \text { Male } & \text { Female } & \\ & \mathrm{n}=28 ; 46.7 \% & \mathrm{n}=32 ; 53.3 \% & 60(100 \%) \\ \text { Age } & 47 \pm 10.4 & 51 \pm 17.6 & \\ & \text { Group II (with no Demodex mites) } & \\ \text { Sex } & \text { Male } & \text { Female } & \\ & \mathrm{n}=24 ; 40.0 \% & \mathrm{n}=36 ; 60.0 \% & 60(100 \%) \\ \text { Age } & 41 \pm 6.3 & 33 \pm 2.9 & \\ & \text { Group III (healthy people) } & \\ \text { Sex } & \text { Male } & \text { Female } & 72(100 \%) \\ & \mathrm{n}=30 ; 41.7 \% & \mathrm{n}=42 ; 58.3 \% & \\ \text { Age } & 26 \pm 1.4 & 31 \pm 3.1 & \end{array}$

$(\mathrm{P}=0.001)$. All cases of mites' detection were recorded with the following predisposing factors: emotional stress $(n=60$; $100 \%)$, inadequate nutrition $(n=24 ; 40 \%)$, exacerbation of concomitant diseases $(n=2$; $3.3 \%)$. There were no statistical differences in the exacerbations of diseases, depending on the season.

In the comparison of the duration of the rosacea in patients of groups I and II significant difference was found $(\mathrm{P}<0.01)$. The presence of Demodex mites in rosacea promotes a longer course of the condition of I group patients (1-5 years duration of rosacea was observed in 10 patients (16.7\%); 44 patients $(73.3 \%)$ had a more than 5 years duration of rosacea). At the same time, in the II group 24 patients (40\%) had a less than one year duration of rosacea, the same number of patients had 1-5 years (24; 40\%) and only 12 patients had more than 5 years of rosacea duration $(20 \%)$.

Comparing the frequency of rosacea recurrence between patients of groups I and II, a statistically significant difference was revealed $(\mathrm{P}<0.05)$. In patients with absence of Demodex mites, in most cases the condition recurred only once $(20 ; 33.3 \%)$, whereas in group I the highest number of patients had $1-3$ recurrence a year $(30 ; 50 \%)$. Thus, Demodex mites complicate the clinical picture of the condition and contribute to a more frequent recurrence of the process.

\section{Evaluation of the Dermatology Quality of Life Index}

When comparing the average indices of the dermatology life quality index, a statistically significant difference was revealed. In Group I patients, the mean value of the index was $12.5 \pm 4.5(\mathrm{~min}=5.0, \max =19.0)$. In Group II patients, the mean value of the dermatology life quality index was $8.0 \pm 3.4$ $(\min =2.0 ; \max =19.0),(P<0.05)$. The average value of the dermatology life quality index in the presence of Demodex brevis is 10.5. In the presence of Demodex folliculo- 
rum and the combined cases of simultaneous detection of two mites species - 15.5 and 13.5 , respectively.

\section{Clinical picture of patients with rosacea}

The distribution of patients by severity of rosacea is presented in Table 2 .

\section{Determination of species affiliation of Demodex mites}

The greatest number of rosacea cases in Group I patients was associated with parasitizing Demodex folliculorum ( $\mathrm{n}=40$; $66.7 \%$ ), Demodex brevis was found in 14 patients $(23.3 \%)$; both mites were found in 6 patients (in $10.0 \%$ of cases). When examining 72 healthy people by light microscopy of scrapes, Demodex mites were detected in six cases $(n=6 ; 2.8 \%)$, in the remaining 66 cases $(97.2 \%)$, mites failed to identify. In determining the species of Demodex mites in healthy people, the parasitism of Demodex brevis was revealed in all 6 cases. Demodex folliculorum $(n=40 ; 66.7 \%)$ is statistically significant in the structure of species affiliation in patients with rosacea. The number of detection cases of this species of mite prevails over the frequency of detection of Demodex brevis and associated parasitism by two species of mite.

A further study showed that Demodex folliculorum accompanies heavier forms of the condition (pustulous and infiltrativeproductive forms of rosacea) $(n=16 ; 26.7 \%$ and $n=22 ; 36.7 \%$, respectively). Combined cases of simultaneous detection of two species of mites (Demodex folliculorum and Demodex brevis) on the scrapes of facial skin by light microscopy also correlated with severe forms of rosacea $(n=4 ; 6.7 \%$ and $n=2 ; 3.3 \%$, respectively).

\section{Comparative analysis of diagnostic methods for detecting Demodex mites}

In this step we compared all patients with rosacea included those, in which cases Demodex mites were revealed by only one method. To assess the validity of the confocal laser scanning in vivo microscopy method, the survey was conducted in all three groups of subjects. A comparative study was performed on the effectiveness of confocal laser scanning in vivo microscopy and scrape methods followed by microscopic study. The data obtained are presented in Table 3.

Positive results for the detection of Demodex mites by the method of light microscopy scrapes were 60 patients with rosacea $(28.3 \%)$, confocal laser scanning in vivo microscopy - 80 patients $(37.7 \%)$. Using confocal laser scanning in vivo microscopy, it was also possible to identify Demodex mites in healthy people $(\mathrm{n}=12$; $5.7 \%$ ), and in the light microscopy of scrapes of Demodex mites in the number of 5 individuals per $1 \mathrm{~cm}^{2}$, only 6 healthy persons $(n=6 ; 2.8 \%)$; in the remaining 66 healthy people $(31.2 \%)$, the light microscopy of the scrapes was negative.

\section{Quantification of Demodex mites}

As a result of the study, we found that it is difficult to detect the mite by light microscopy of scrape per $1 \mathrm{~cm}^{2}$ of skin (Table 4).

Table 2. Distribution of patients with rosacea I and II groups according to the severity of the condition.

\begin{tabular}{lccc} 
Form of the condition & Group I ( $\mathbf{n} ; \%)$ & Group II (n; \%) & Total (n) \\
Erythematous-telangiectatic & $6 ; 10$ & $28 ; 46.7^{*}$ & 34 \\
Papular & $8 ; 13.3$ & $22 ; 36.7$ & 30 \\
\hline Pustulous & $22 ; 36.7^{*}$ & $10 ; 16.6$ & 32 \\
Infiltrative-productive & $24 ; 40^{*}$ & 0 & 24 \\
\hline Total & $60 ; 100$ & $60 ; 100$ & 120 \\
\hline
\end{tabular}

Table 3. Comparative analysis of study methods for the presence of Demodex mites in diagnostically significant amounts.

\begin{tabular}{|c|c|c|c|c|}
\hline Method & $\begin{array}{c}\text { Identification of } \\
\text { Demodex mites } \\
\text { ( }>5 \text { individuals per } 1 \mathrm{~cm}^{2} \text { ) }\end{array}$ & $\begin{array}{l}\text { Patients diagnosed } \\
\text { with rosacea } \\
(\mathrm{n}=140 ; 66 \%)\end{array}$ & $\begin{array}{l}\text { Healthy people } \\
(\mathrm{n}=72 ; 34 \%)\end{array}$ & $\begin{array}{c}\text { Total } \\
(\mathrm{n}=212 ; 100 \%)\end{array}$ \\
\hline Scrape with subsequent & + & $60 ; 28.3 \%$ & $6 ; 2.8 \%$ & $66 ; 31.1 \%$ \\
\hline light microscopy & - & $80 ; 37.7 \%$ & $66 ; 31.2 \%$ & $146 ; 68.9 \%$ \\
\hline Method & $\begin{array}{c}\text { Identification of } \\
\text { Demodex mites } \\
\left(>5 \text { individuals per } 1 \mathrm{~cm}^{2} \text { ) }\right.\end{array}$ & $\begin{array}{l}\text { Patients diagnosed } \\
\text { with rosacea } \\
(n=140 ; 66 \%)\end{array}$ & $\begin{array}{c}\text { Healthy people } \\
(\mathrm{n}=72 ; 34 \%)\end{array}$ & $\begin{array}{c}\text { People } \\
(n=212 ; 100 \%)\end{array}$ \\
\hline Confocal laser scanning & + & $80 ; 37.7 \%$ & $12 ; 5.7 \%$ & $92 ; 43.4 \%$ \\
\hline in vivo microscopy & - & $60 ; 28.3 \%$ & $60 ; 28.3 \%$ & $88: 56.6 \%$ \\
\hline
\end{tabular}

Table 4. Detection of Demodex mites by the method of light microscopy of scrapes.

\begin{tabular}{|c|c|c|c|c|c|c|}
\hline $\begin{array}{l}\text { Patients with } \\
\text { rosacea with } \\
\text { the presence }\end{array}$ & \multicolumn{2}{|c|}{$\begin{array}{l}\text { Patients with rosacea } \\
\text { with no Demodex mites } \\
\text { (n; \%) }\end{array}$} & \multicolumn{3}{|c|}{$\begin{array}{c}\text { Healthy } \\
\text { people }(\mathrm{n} ; \%)\end{array}$} & \multirow[t]{2}{*}{$\begin{array}{l}\text { Total } \\
(\mathrm{n} ; \%)\end{array}$} \\
\hline $\begin{array}{l}\text { Pesence of } \\
\text { Demodex mites } \\
>5 / \mathrm{cm}^{2}\end{array}$ & $\begin{array}{c}\text { Presence of } \\
\text { Demodex mites } \\
<5 / \mathrm{cm}^{2}\end{array}$ & $\begin{array}{l}\text { Absence of } \\
\text { Demodex mites }\end{array}$ & $\begin{array}{l}\text { Presence of } \\
\text { Demodex mites } \\
>5 / \mathrm{cm}^{2}\end{array}$ & $\begin{array}{c}\text { Presence of } \\
\text { Demodex mites } \\
>5 / \mathrm{cm}^{2}\end{array}$ & $\begin{array}{l}\text { Absence of } \\
\text { Demodex mites }\end{array}$ & \\
\hline $60 ; 28.3$ & $22 ; 10.4$ & $58 ; 27.4$ & $2 ; 0.9$ & $4 ; 1.9$ & $66 ; 31.1$ & $212 ; 100$ \\
\hline $60 ; 28.3$ & \multicolumn{2}{|c|}{$80 ; 37.8$} & & $72 ; 33.9$ & & \\
\hline
\end{tabular}


Demodex mites in a diagnostically significant amount ( $>5$ individuals per $1 \mathrm{~cm}^{2}$ ) were revealed in 60 patients with rosacea (28.3\%), included in group I. In 80 patients with rosacea $(37.8 \%)$ with Demodex mites were detected in an amount of less than 5 individuals per $1 \mathrm{~cm}^{2}$ or were absent altogether with a developed clinical picture of the condition.

In the examination of healthy people, 66 people (31.1\%) had a negative analysis for the presence of Demodex mites, while Demodex mites were found in a diagnostic amount (more than 5 individuals per $\mathrm{cm}^{2}$ ) in two subjects $(0.9 \%)$, in four respondents, the mites were found in an amount of less than 5 individuals per $\mathrm{cm}^{2}(1.9 \%)$. Demodex mites in an amount of $<5$ individuals per 1 $\mathrm{cm}^{2}$ were detected much more often $(\mathrm{n}=80$; $37.8 \%$, respectively). While study the same respondents using confocal laser scanning in vivo microscopy, the following data were obtained (Table 5). When analyzing the data in Table 6 attention is drawn to the fact that there were no $<5$ mites per $\mathrm{cm}^{2}$ in any case By using confocal laser scanning in vivo microscopy, Demodex mites were found in patients with rosacea $(n=80,37.8 \%)$ and in healthy people in a larger number of cases $(\mathrm{n}=12 ; 5.7 \%)$.

Using a confocal laser scanning in vivo microscope allowed determining the average size of Demodex mites. When determining the size of mites from 100 to $200 \mu \mathrm{m}$, it was believed that in this case Demodex brevis was observed, while the average length of the mite was $125 \mu \mathrm{m}$; from 200 to 400 $\mu \mathrm{m}-$ Demodex folliculorum with an average length of $293 \mu \mathrm{m}$. The average size of the width of Demodex mites was $24 \mu \mathrm{m}$.

When measuring the size of the follicular aperture and estimating their number per unit area, for which a randomly chosen site of $25 \mathrm{~mm}^{2}$ was taken, statistically significant differences between the three groups were revealed $(\mathrm{P}<0.01)$. It was established that the size of the follicular aperture and their number per unit area in all three groups differ significantly. It was found that the largest size of the mouths of the hair follicles and the excretory ducts of the sebaceous glands were patients of group I with Demodex mites $(0.125 \pm 25 \mu \mathrm{m})$, whereas in group II the size of the hair follicles and sebaceous glands was $0.89 \pm 32 \mu \mathrm{m}$, in group III $-0.072 \pm 29 \mu \mathrm{m}$.

Patients of group I had the highest number of follicles and excretory ducts of sebaceous glands in study groups per $25 \mathrm{~mm}^{2}$ $(324 \pm 11)$, in group II $-114 \pm 6$ and in group III - $28 \pm 7$.

\section{Antiparasitic therapy in patients with rosacea}

After obtaining a positive result of the study for the presence of Demodex mites, the patients were randomized according to treatment regimens into two equal subgroups (A and B, respectively) for 30 people each. Patients enrolled in subgroup A received only external therapy with a drug containing $1 \%$ ivermectin in the form of a cream 1 time per day for 30 days. Patients enrolled in subgroup B received a drug containing $250 \mathrm{mg}$ of metronidazole systemically 2 times a day, externally $1 \%$ metronidazole in the form of a gel 1 time per day for 30 days. A repeat visit of the patients took place after 30 days of continuous therapy. Subjectively, treatment regimens of patients were well tolerated, no side effects were noted, no patient was excluded from the study. When comparing the efficacy of the therapy, it was found that statistically significantly more Demodex mites were found after treatment with confocal laser scanning in vivo microscopy $(\mathrm{P} \leq 0.05)$ (Table 7). For a comparative evaluation of the effectiveness of treatment methods in subgroups A and B, the clinical picture was compared in the next stage in patients with rosacea associated with Demodex mites before and after treatment.

Complaints of patients before and after the treatment are given in Table 8 .

As can be seen from Table 8 , after treatment statistically reliably decreased complaints of patients on rashes, burning sensa- tion, pain, pruritus, and rubeosis. Moreover, in patients with subgroup A, complaints of greasy lusters of skin gloss decreased, which is an additional advantage of topical therapy. An objective analysis of the clinical picture of patients with rosacea associated with Demodex mites after the therapy showed that the following morphological elements in subgroups A and B significantly regressed: papules, pustules, excoriation $(\mathrm{P} \leq 0.05)$ compared with the original data.

Analysis of the clinical picture showed a positive dynamics of therapy, which manifested itself in a significant decrease in the number of morphological elements characterizing the severity of inflammation $(\mathrm{P} \leq 0.05)$. The effectiveness of the therapy was confirmed by a reduction in subjective complaints of patients after the treatment, and patients who received only external therapy had no complaints of a feeling of lusters of skin and the appearance of greasy lusters, which is an additional advantage. Thus, clinical observations demonstrated a lack of superiority in combined antiparasitic therapy using a systemic drug compared to external therapy using a preparation containing $1 \%$ ivertmectin as a cream, as confirmed by statistical analysis.

\section{Discussion}

Association of Demodex mites identification with predisposing factors such as emotional stress, inadequate nutrition, exacerbation of concomitant diseases are correlated with the data of already available studies, in which the role of trigger factors in the development of demodecosis is discussed.9${ }^{12}$ In addition, the effect of heat, cold, spicy food on the development of rosacea wasdetailed in a study by Aubdool. ${ }^{13}$

Our findings confirm the hypothesis of Turgut Erdemir et al., that the Demodex mites affect the severity of the disease and contribute to the progression of the pathological process. In addition, the authors

Table 5. Detection of Demodex mites by confocal laser scanning in vivo microscopy.

\begin{tabular}{|c|c|c|c|c|}
\hline $\begin{array}{l}\text { Patients with } \\
\text { rosacea with } \\
\text { the presence } \\
\text { of Demodex mites } \\
\text { (n; \%) } \\
\text { Presence of } \\
\text { Demodex mites }>5 / \mathrm{cm}^{2}\end{array}$ & $\begin{array}{l}\text { Patients with rosacea } \\
\text { with no Demodex mites } \\
\text { (n; \%) }\end{array}$ & $\begin{array}{c}\text { Presence of } \\
\text { Demodex } \text { mites }>5 / \mathrm{cm}^{2}\end{array}$ & $\begin{array}{c}\text { Absence of } \\
\text { Demodex mites }\end{array}$ & $\begin{array}{c}\text { Total } \\
(\mathrm{n} ; \%)\end{array}$ \\
\hline $80 ; 37.8$ & $60 ; 28.3$ & $12 ; 5.7$ & $60 ; 28.3$ & $212 ; 100$ \\
\hline $80 ; 37.8$ & $60 ; 28.3$ & \multicolumn{2}{|c|}{$72 ; 34$} & \\
\hline
\end{tabular}


have proved that the density of mites increases depending on the severity of the disease. ${ }^{14}$

The effect of rosacea on the life and social activity of patients is still debated. ${ }^{15}$ In a meta-analysis of Bewley et al., which included 1,624 patients diagnosed with rosacea, 26.4\% complained of anxiety and depression, and $43 \%$ had a quality of life violation. $62 \%$ of respondents indicated that rosacea affected their work and social life, and $26.1 \%$ began to avoid social contacts because of the disease. ${ }^{16}$ When comparing the average indicators of the Dermatology Quality of Life Index, it was revealed that in patients of group I the condition has a very strong effect on the life of the patient, in patients in group II; rosacea had a moderate effect on the life of patients. Rosacea, complicated by the presence of Demodex brevis, has a moderate effect on life of patients. In the presence of Demodex folliculorum and combined cases of simultaneous detection of two species of mites, the condition greatly affects the life of patients. In patients with the presence of Demodex folliculorum, the dermatology life quality index is statistically significantly different from the index of patients with Demodex brevis $(\mathrm{P}<0.05)$, which is logically logical, since we estab- lished that Demodex folliculorum is found in patients with heavier forms of diseases.

The detection of Demodex mites is not only statistically more significant in patients with rosacea than in the rest of the population, ${ }^{17}$ but also as can be seen from the Table 2, Demodex mites were more often found in patients with more severe clinical forms of rosacea (pustulous, infiltrative-productive forms).

Such pathological formations as deep papulopustulous elements and nodes, facial skin erythema, greasy lustre are more pronounced in patients of group I, which again confirms that the presence of Demodex mites predisposes to the emergence of acute inflammatory morphological elements, contributing to the development of more severe clinical forms of diseases. Our results coincide with the results of a study by Moravvej et al., where it was proved that mites play a role in the development of rosacea, stimulate the formation of an inflammatory process, which subsequently leads to tissue damage and the formation of telangiectasias. ${ }^{18}$

The data obtained in determining the species belonging to Demodex mites show that Demodex folliculorum is more often detected in the analyses than Demodex bre-

Table 6. Analysis of the clinical picture of patients with rosacea associated with Demodex mites, before and after the therapy.

\begin{tabular}{|c|c|c|c|c|}
\hline \multirow[t]{2}{*}{ Elements } & \multicolumn{2}{|c|}{ Sub-group $\mathbf{A}$} & \multicolumn{2}{|c|}{ Sub-group B } \\
\hline & $\begin{array}{l}\text { Before } \\
\text { treatment }\end{array}$ & $\begin{array}{c}\text { After } \\
\text { treatment }\end{array}$ & $\begin{array}{l}\text { Before } \\
\text { treatment }\end{array}$ & $\begin{array}{c}\text { After } \\
\text { treatment }\end{array}$ \\
\hline Papules & $100^{*}$ & 43 & $74^{*}$ & 48 \\
\hline Pustules & $82^{*}$ & 30 & 63 & 42 \\
\hline Open comedons & $87^{*}$ & 42 & $87^{*}$ & 47 \\
\hline Milium & $60 *$ & 23 & $41^{*}$ & 14 \\
\hline Teleangiectasias & $41^{*}$ & 14 & $56^{*}$ & 12 \\
\hline Perifocal erythema & $56^{*}$ & 20 & $75^{*}$ & 36 \\
\hline Excoriations & $45^{*}$ & 11 & $68^{*}$ & 21 \\
\hline Pigmentation & 74 & 53 & $90^{*}$ & 63 \\
\hline Greasy lusters of skin & $65^{*}$ & 25 & 72 & 56 \\
\hline
\end{tabular}

vis and Demodex brevis is detected more often than the combined cases are recorded. Demodex folliculorum is statistically significantly more common in patients than simultaneous parasitization of two species of mites $(\mathrm{P}<0.01)$. This suggests that in the absence of a clinical picture of rosacea, it is significantly more significant that Demodex mites' species will not be found in scrape $(\mathrm{P}<0.01)$. In studies by Erbağci et al. (1998), Divani et al. (2009) and GonzalezHinojosa et al. (2018) demonstrated that statistically significantly more people in the clinical manifestations of rosacea will be found to have Demodex mite than in healthy volunteers. ${ }^{19-21}$

Comparing the results obtained by light microscopy and confocal laser scanning in vivo microscopy in patients with rosacea and healthy people, in more cases Demodex mites are detected by confocal laser scanning in vivo microscopy, whereas scrape in these patients were negative. The obtained data demonstrate not only high information content of the confocal laser scanning in vivo microscopy method, but also its superiority over microscopic diagnostics. This was also demonstrated in studies by Gonzalez et al. and Rajadhyaksha et al. ${ }^{22,23}$

In the examination of healthy people by light microscopy, Demodex mites were detected in 6 cases $(2.8 \%)$. Given the ability of the mites to move over the surface of the skin at a speed of $8-16 \mathrm{~mm} / \mathrm{h},{ }^{9,10}$ as well as random selection of the study site, this fact does not prove the absence of mites. Given the species belonging to Demodex mites in healthy people (all had Demodex brevis); the absence of a clinical picture of the condition confirms that this species belongs to saprophyte of facial skin. By using confocal laser scanning in vivo microscopy, Demodex mites were defined as rounded or long conical formations in the mouths of the hair and sebaceous glands with the presence of peripheral contouring in the number from one to 25 individuals (an average of 3.37). Sattler et al. showed the possibility of using confocal laser scanning in vivo microscopy to determine Demodex mites and described

Table 7. Comparative detection table of Demodex mites after the therapy in subgroups A and B.

\begin{tabular}{|c|c|c|c|c|}
\hline & \multicolumn{2}{|c|}{$\begin{array}{l}\text { Scrape of the skin, squeezing the contents } \\
\text { of the sebaceous glands followed } \\
\text { by microscopy }\end{array}$} & \multicolumn{2}{|c|}{$\begin{array}{l}\text { Confocal laser scanning } \\
\text { in vivo microscopy }\end{array}$} \\
\hline & $\begin{array}{l}\text { Presence of } \\
\text { Demodex mites } \\
\text { (n; \%) }\end{array}$ & $\begin{array}{l}\text { Absence of } \\
\text { Demodex mites } \\
(\mathrm{n} ; \%)\end{array}$ & $\begin{array}{l}\text { Presence of } \\
\text { Demodex mites } \\
\quad(\mathrm{n} ; \%)\end{array}$ & $\begin{array}{l}\text { Absence of } \\
\text { Demodex mites } \\
\text { (n; \%) }\end{array}$ \\
\hline Sub-group A & $2 ; 3.3$ & $28 ; 46.7$ & $4 ; 6.7^{*}$ & $26 ; 43.3$ \\
\hline Sub-group B & $4 ; 6.7$ & $26 ; 43.3$ & $10 ; 16.7^{*}$ & $20 ; 33.3$ \\
\hline
\end{tabular}


them as rounded and long conical structures. $^{7}$ A comparison of the effectiveness of the performed therapy once again demonstrates the superiority of confocal laser scanning in vivo microscopy over the subsequent light microscopy (Table 7). When analyzing the data obtained in Table 7 , it can be stated that both methods of treatment had high antiparasitic efficacy. Considering the fact that there was no statistically significant difference in negative analyzes for the presence of Demodex mites in both laboratory and instrumental diagnostics, we can speak of a high antiparasitic efficacy of a topical medicine containing $1 \%$ ivertmectin in comparison with combined treatment with the systemic drug metronidazole and a topical agent containing $1 \%$ metronidazole. The relevance of ivermectin for the treatment of rosacea has been shown in a study by Cardwell et al. ${ }^{24}$ When comparing 1\% ivermectin and $0,75 \%$ metronidazole, ivermectin showed greater efficacy. This was expressed in the quality of life of patients, reducing the number of inflammatory elements and the absence of side effects. ${ }^{24}$

Analysis of the clinical picture showed a positive dynamic of therapy, which manifested itself in a significant decrease in the number of morphological elements characterizing the severity of inflammation $(\mathrm{P}<0.05)$. The effectiveness of the therapy was confirmed by a reduction in subjective complaints of patients after the treatment, and patients who received only external therapy had no complaints of a feeling of lustre of skin and the appearance of a greasy lustre, which is an additional advantage. Thus, clinical observations demonstrated a lack of superiority in combined antiparasitic therapy using a systemic drug compared to external therapy using a preparation containing $1 \%$ ivertmectin as a cream, as confirmed by statistical analysis. Stein et al. showed that after 12 weeks of ivermectin treatment, the skin of patients was defined as clean or almost clean. There was a significant reduction in the percentage of inflammatory lesions in the ivermectin treatment group. The results of the study showed that $1 \%$ ivermectin is an effective and safe treatment for inflammatory lesions in patients with rosacea. ${ }^{25}$

\section{Conclusions}

As a conclusion, the following is stated:

i) Demodex mites complicate the clinical picture and the course of rosacea. When analyzing the clinical picture and the course of the condition in patients with rosacea associated with Demodex mites, it is established that Demodex mites, promote the development of acute-inflammatory morphological elements (deep papular, pustulous elements, nodes, perifocal erythema), increase the duration of the condition (more than 5 years, $\mathrm{P}<0.01$ ) and the probability of recurrence (from 1 to 3 relapses in $39.5 \%$ of patients, $\mathrm{P}<0.05$ ), resulting in a decrease in the quality of life of patients (dermatology life quality index $12.5 \pm 4.5, \mathrm{P}<0.05$ ). The severity of the clinical manifestations of rosacea does not depend on the number of individuals detected by the method of light microscopy of scrapes of facial skin. The most significant factors predisposing to the development of complications are: morphofunctional characteristics of the skin (high greasiness, reduced moisture, alkaline $\mathrm{pH}$ shift, and larger pore size), emotional impact, including stress, inadequate nutrition and exacerbation of concomitant diseases.

ii) In patients with severe manifestations of the condition (pustulous and infil-

Table 8. Comparison of subjective complaints of patients with rosacea associated with Demodex mites before and after treatment.

\begin{tabular}{|c|c|c|c|c|}
\hline \multirow[t]{2}{*}{ Complaints } & \multicolumn{2}{|c|}{ Sub-group A } & \multicolumn{2}{|c|}{ Sub-group B } \\
\hline & $\begin{array}{l}\text { Before } \\
\text { treatment }\end{array}$ & $\begin{array}{c}\text { After } \\
\text { treatment }\end{array}$ & $\begin{array}{l}\text { Before } \\
\text { treatment }\end{array}$ & $\begin{array}{c}\text { After } \\
\text { treatment }\end{array}$ \\
\hline Rashes & $100 \%$ & $70 \% *$ & $100 \%$ & $65 \% *$ \\
\hline Pain & $68 \%$ & $34 \% *$ & $75 \%$ & $30 \% *$ \\
\hline Burning sensation & $59 \%$ & $40 \%$ & $64 \%$ & $30 \% *$ \\
\hline Rubeosis & $69 \%$ & $40 \% *$ & $70 \%$ & $40 \% *$ \\
\hline Pruritus & $70 \%$ & $20 \% *$ & $54 \%$ & $10 \% *$ \\
\hline Chromatosis & $42 \%$ & $25 \%$ & $36 \%$ & $17 \%$ \\
\hline Presence of crusts/excoriations & $45 \%$ & $30 \%$ & $39 \%$ & $20 \%$ \\
\hline Starburst veins & $60 \%$ & $40 \%$ & $58 \%$ & $40 \%$ \\
\hline Greasy lusters of skin & $70 \%$ & $40 \% *$ & $65 \%$ & $40 \%$ \\
\hline
\end{tabular}

\section{References}

1. Lazaridou E, Giannopoulou C, Fotiadou C, et al. The potential role of microorganisms in the development of rosacea. J Dtsch Dermatol Ges 2011;9:21-5.

2. Schommer NN, Gallo RL. Structure and function of the human skin microbiome. Trends Microbiol 2013;21:660-8.

3. Norn MS. Demodex folliculorum. Incidence, regional distribution, patho- 
genicity. Dan Med Bull 1971;18:14-7.

4. Rufli T, Mumcuoglu Y. The hair follicle with Demodex folliculorum and Demodex brevis mites: Biology and medical importance. Dermatologica 1981;162:1-11.

5. Fujiwara, S, Okubo Y, Irisawa R, Tsuboi R. Rosaceiform dermatitis associated with topical tacrolimus treatment. J Am Acad Dermatol 2010;62: 1050-2.

6. Akbulatova LK. The pathogenic role of Demodex mites and the clinical form of demodicosis in human being. Vestn Dermatol Venerol 1963;40:57-61.

7. Sattler EC, Maier T, Hoffmann VS, et al. Noninvasive in vivo detection and quantification of Demodex mites by confocal laser scanning microscopy. $\mathrm{Br}$ J Dermatol 2012;167:1042-7.

8. Wilkin J, Dahl M, Detmar M, et al. Standard classification of rosacea: Report of the National Rosacea Society Expert Committee on the Classification and Staging of Rosacea. J Am Acad Dermatol 2002;4:584-7.

9. Hoekzema R, Hulsebosch HJ, Bos JD. Demodecosis or rosacea: what did we treat? Br J Dermatol 1995;133:294-9.

10. Demmler M, de Kaspar HM, Mohring C, Klauss V. Blepharitis. Demodex folliculorum, associated pathogen spectrum and specific therapy. Ophthalmologe 1997;94:191-6.
11. Wilkin JK. Oral thermal-induced flushing in erythematotelangiectatic rosacea. J Invest Dermatol 1981;76:15-8.

12. Bernstein JE. Rosacea flushing. Int $J$ Dermatol 1982;21:24.

13. Aubdool AA, Brain SD. Neurovascular aspects of skin neurogenic inflammation. J Investig Dermatol Symp Proc 2011;15:33-9.

14. Turgut Erdemir A, Gurel MS, Koku Aksu AE, et al. Demodex mites in acne rosacea: reflectance confocal microscopic study. Australas J Dermatol 2017;58:e26-30.

15. Gallo RL, Granstein RD, Kang S, et al. Rosacea comorbidities and future research: The 2017 update by the National Rosacea Society Expert Committee. J Am Acad Dermatol 2018;78:167:70.

16. Bewley A, Fowler J, Schöfer H, et al. Erythema of rosacea impairs healthrelated quality of life: Results of a meta-analysis. Dermatol Ther 2016;6:237-47.

17. Roihu T, Kariniemi AL. Demodex mites in acne rosacea. J Cutan Pathol 1998;25:550-2.

18. Moravvej H, Dehghan-Mangabadi M, Abbasian M-R, Meshkat-Razavi G. Association of Rosacea with Demodicosis. Arch Iranian Med 2007;10:199-203.

19. Erbağci Z, Ozgöztaşi O. The signifi- cance of Demodex folliculorum density in rosacea. Int J Dermatol 1998;37:4215.

20. Divani S, Barpakis K, Kapsalas D. Chronic blepharitis caused by Demodex folliculorum mites. Cytopathology 2009;20:343-4.

21. Gonzalez-Hinojosa D, Jaime-Villalonga A, Aguilar-Montes G, LammogliaOrdiales L. Demodex and rosacea: is there a relationship? Indian J Ophtalmol 2018;66:36-8.

22. Gonzalez S, Rajadhyaksha M, Anderson RR. Non-invasive (real-time) imaging of histologic margins of a proliferative skin lesion. In vivo. J Invest Dermatol 1998;111:538-9.

23. Rajadhyaksha M, Grossman M, Esterowitz D, et al. In vivo confocal scanning laser microscopy of human skin: melanin provides strong contrast. J Invest Dermatol 1995;104:946-52.

24. Cardwell LA, Alinia H, Tuchayi SM, Feldman SR. New developments in the treatment of rosacea - role of oncedaily ivermectin cream. Clin Cosmet Investig Dermatol 2016;9:71-7.

25. Stein L, Kircik L, Fowler J, et al. Efficacy and safety of ivermectin 1\% cream in treatment of papulopustular rosacea: results of two randomized, double-blind, vehicle-controlled pivotal studies. J Drugs Dermatol 2014;13:31623. 